\title{
Mesiodistal root angulation of permanent teeth in children with mixed dentition and normal occlusion
}

\author{
Flávia A. S. JESUINO', Luciane R. COSTA², José VALLADARES-NETO³
}

1- DDS, MSc, PhD, Clinical Instructor of Orthodontics, Department of Prevention and Oral Rehabilitation, Dental School, Federal University of Goiás, Goiânia, GO, Brazil.

2- DDS, MSc, PhD, Associate Professor of Pediatric Dentistry, Department of Prevention and Oral Rehabilitation, Dental School, Federal University of Goiás, Goiânia, GO, Brazil.

3- DDS, MSc, Assistant Professor of Orthodontics, Department of Prevention and Oral Rehabilitation, Dental School, Federal University of Goiás, Goiânia, GO, Brazil.

Corresponding address: Luciane R. Costa - Primeira Avenida, sem número, Setor Universitário - Goiânia, GO - Brasil - 74605-220 - e-mail: Isucasas@ odonto.ufg.br - Phone: 556232096047 - Fax: 556232096065

Received: March 23, 2009 - Modification: August 01, 2009 - Accepted: October 22, 2009

\section{ABSTRACT}

O bjective: There is little information regarding the mesiodistal angulation of permanent teeth in mixed dentition. The aim of this study was to evaluate mesiodistal root angulation of permanent incisors, canines and first molars of 100 Brazilian children, using a new horizontal reference plane based on the midpoint of the intercuspation of primary canines and permanent first molars in panoramic radiographs during the mixed-dentition phase. Material and Methods: Children were equally divided between the genders with a mean age of 8.9 years $(S D=0.76)$, normal occlusion and no eruptive disturbances. Results: The angulation of the permanent maxillary first molars was close to the vertical, whereas the mandibular molars presented approximately 25 degrees of distal root angulation. The maxillary canines were the most distally angulated teeth, whereas the permanent mandibular canines were vertically positioned. The evaluation of the anterior maxillary area showed vertical position of permanent lateral, and central incisors with a slight distal angulation, whereas the permanent mandibular incisors tended to a mesial radicular convergence. Conclusions: The proposed reference line could be useful in mixed dentition root angulation evaluation; there was a slight asymmetry in the mesiodistal angulation among homologous teeth, and also a small variation between the male and the female groups, but no difference between 8-and 10-year-old children.

Key words: Panoramic radiography. Mixed dentition. Interceptive orthodontics. Dental occlusion.

\section{INTRODUCTION}

Most of the studies relating to the analysis of mixed dentition use plaster casts to evaluate the available space for the eruption of permanent teeth $^{1,7,14}$. Plaster cast analysis cannot, however, determine the position of unerupted teeth, even for ideal space conditions. Radiographs, on the other hand, allow for the simultaneous evaluation of the tooth diameter ${ }^{9}$ and intraosseous position ${ }^{9,18}$.

There are few available radiographic studies aimed at predicting the space conditions from mixed to permanent dentition. Most of these studies are restricted to the evaluation of the ectopic eruption of the maxillary canines ${ }^{13,18}$. Schindel and Duffy ${ }^{13}$ (2007) concluded that there is a close relationship between the transverse maxillary discrepancy and potentially impacted canines, which can be identified by panoramic radiographs.

Mesiodistal root angulation alterations of the maxillary and mandibular incisors may significantly change the molar relationship ${ }^{12}$. The angulations of permanent first molars, incisors and canines on panoramic radiographs could provide data relating to the tooth eruption in mixed dentition. Several studies $^{12,13,18}$ describe the angulation alterations of incisors and impacted canines. Even though there are no studies on mesiodistal root angulation in mixed dentition with normal occlusion and wellbalanced faces, reference values for this angulation are required for professional knowledge. The aim of this study was to evaluate mesiodistal root 
angulation of permanent incisors, canines and first molars of a Brazilian child sample with mixed dentition, using the horizontal reference line drawn on a panoramic radiograph.

\section{MATERIAL AND METHODS}

\section{Patients}

This study was approved by the Research Ethics Committee of the Federal University of Goiás (UFG), Brazil (Protocol \#051/2007). All the children had the informed consent form signed by their lawful representative. One hundred children, with a mean age of 8.9 years $(S D=0.76)$, were chosen from the dental school clinics of the (UFG), private dental offices, and public and private schools. The inclusion criteria were: middle mixed dentition stage, Class I occlusion with no tooth crowding above $2 \mathrm{~mm}$ or history of orthodontic treatment, and a well-balanced face, without skeletal disharmony. Children with syndrome aspects, skull or dentofacial anomalies or early loss of primary teeth were excluded. Fifty girls and fifty boys with ages varying from 8 years to 10 years and 11 months were evaluated. The sample was subdivided into three groups according to their age: eight $(n=49)$, nine $(n=37)$ and ten $(n=14)$ years old. The aim was to verify any change in mesiodistal root angulation influenced by canine eruption during the "ugly duckling stage"2. The comparison was done only between the youngest (eight years old) and oldest (ten years old) groups.

\section{Data collection}

The children were first chosen by visual inspection by the researcher while they were standing, looking straight at the horizon and maintaining a natural head position ${ }^{6}$. The child was asked to smile and if there was no visible malocclusion, the dental occlusion was clinically examined, the cheeks being moved with a wooden spatula. The researcher evaluated the canine relationship and other deviations. All children had their panoramic radiograph taken in the same radiological clinic by Gendex Orthoralix 9200 DDE apparatus (Gendex Dental Systems, Des Plaines, IL, USA). The patients were positioned in a standard manner, with the teeth in maximum intercuspation, the occlusal plane kept horizontal and the facial midline perpendicular to it. The pictures were processed by an automatic processing machine (Xtec-Revell, São Paulo, SP, Brazil). Each radiograph was checked and those considered of poor quality were repeated or discarded.

\section{Analysis of the Panoramic Radiograph}

All panoramic radiographs were evaluated by the same examiner in a dark room, using a light box. The anatomic structures from the panoramic radiograph were drawn on $0.07-\mathrm{mm}$-thick acetate paper $(29.5 \times 15.0 \mathrm{~cm})$ with a 0.05 pencil point. The drawing included the orbital lower contour, the mandible's external profile, the contour of the permanent first molars, canines and incisors and crowns of the primary maxillary and mandibular canines. Two reference lines were drawn on both left and right sides, passing at the middle intercuspation point of the first permanent molars and the primary canines. It was decided to use a single reference line for maxillary and mandibular teeth, based on the intercuspation of the teeth (Figure 1). The mental line ${ }^{17}$ was discarded because in the studied sample it was noticed that the mental foramen was clearly visible in less than $20 \%$ of the children.

The long root axis was determined according to Ursi, et al. ${ }^{17}$ (1990). For the single-rooted teeth, the root canal image in its greatest length was selected; for the mandibular molars, the mean of the mesial and distal root canals and another point in the middle of the clinical crown was chosen, and for the maxillary molars, the palatal root image and another point in the middle of the clinical crown were used. The angles of the right and left reference lines and the long axis of the tooth were measured on each radiograph. The measured angle was external, located distally to the midline (Figure 1).

\section{Statistical Analysis}

After assessing the normality of the sample, the Student's t-test for independent samples was selected to compare the mesiodistal root angulations between the genders, right and left lateral teeth and the extreme age ranges in the examined groups ( 8 and 10 years). The calculations were made with the SPSS for Windows v.12.0 software. The level of significance was $5 \%$.

\section{RESULTS}

Method error (ME) was calculated by Dahlberg's formula $M E=\sqrt{\Sigma d^{2} / 2 n}$ ( $d=$ first and second measurements difference, $\mathrm{n}=$ exams measured twice number) to check the intra-examiner error ${ }^{3}$. One week after the first evaluation, the examiner analyzed and measured 40 radiographs again. The greatest error obtained was $1.73^{\circ}$, which was considered small enough to be acceptable.

The mean mesiodistal root angulation for permanent molars, canines and incisors was established separately for the female and male groups (Table 1 ). In the maxilla, the boys had significantly greater angulations than the girls in the following permanent tooth groups: right central incisors, right and left canines. Girls' mandibles were more angulated in the left side than in the region of the central and lateral incisors.

The data obtained in this study were analyzed as follows: for the first maxillary molars, angulation 
close to 90 degrees in a vertical position, whereas the mandibular molars had approximately 25 degrees of distal root angulation; the maxillary canines were the most distally angulated teeth, approximately 66 degrees. The mandibular canines were vertically positioned and the maxillary anterior area exhibited vertical lateral incisors and central incisors with a slight distal angulation. In the mandible, a root convergence to mesial was noticed, which resulted in well aligned crowns with a discrete distal angulation.

Given that the whole population has normal homogeneous characteristics, we sought to define whether homologous permanent teeth had the same angulation (Figure 2). In the maxilla, the incisors and canines were significantly more angulated on the right side. In the mandible, the first molars were significantly more angulated on the left side, and the right lateral incisors were more angulated than the left lateral incisors.

The mesiodistal angulation values were also compared between the youngest (eight years old) and oldest (ten years old) groups and no statistically significant differences were found.

Table 1- Mesiodistal angulation of different teeth in children [mean age 8.9 years \pm standard deviation $(S D=0.76)$ ] with mixed dentition, according to gender

\begin{tabular}{|c|c|c|c|}
\hline \multirow[t]{2}{*}{ Teeth } & \multicolumn{2}{|c|}{ Teeth angulations (degrees), (mean \pm SD) } & \multirow[t]{2}{*}{ P-value } \\
\hline & Male $(n=50)$ & Female $(n=50)$ & \\
\hline \multicolumn{4}{|c|}{ Maxillary arch, right side } \\
\hline First molar & $91.16 \pm 4.71$ & $89.84 \pm 3.95$ & 0.132 \\
\hline Canine & $69.66 \pm 5.95$ & $65.94 \pm 6.93$ & 0.005 \\
\hline Lateral Incisor & $89.82 \pm 7.04$ & $86.98 \pm 7.36$ & 0.052 \\
\hline Central Incisor & $81.32 \pm 6.53$ & $77.74 \pm 5.79$ & 0.005 \\
\hline \multicolumn{4}{|c|}{ Maxillary arch, left side } \\
\hline First molar & $90.82 \pm 4.18$ & $90.16 \pm 3.97$ & 0.420 \\
\hline Canine & $67.10 \pm 5.57$ & $63.74 \pm 7.06$ & 0.010 \\
\hline Lateral Incisor & $87.02 \pm 7.06$ & $85.16 \pm 7.04$ & 0.190 \\
\hline Central Incisor & $78.12 \pm 5.81$ & $76.86 \pm 8.21$ & 0.378 \\
\hline \multicolumn{4}{|c|}{ Mandibular arch, right side } \\
\hline First molar & $75.98 \pm 5.23$ & $75.06 \pm 5.80$ & 0.406 \\
\hline Canine & $86.16 \pm 7.45$ & $83.42 \pm 8.47$ & 0.089 \\
\hline Lateral Incisor & $108.74 \pm 8.60$ & $110.08 \pm 11.14$ & 0.502 \\
\hline Central Incisor & $104.70 \pm 7.68$ & $106.70 \pm 8.96$ & 0.233 \\
\hline \multicolumn{4}{|c|}{ Mandibular arch, left side } \\
\hline First molar & $78.64 \pm 4.88$ & $79.16 \pm 5.45$ & 0.616 \\
\hline Canine & $85.38 \pm 7.38$ & $86.70 \pm 8.82$ & 0.419 \\
\hline Lateral Incisor & $102.42 \pm 6.61$ & $105.74 \pm 9.43$ & 0.044 \\
\hline Central Incisor & $98.44 \pm 5.95$ & $102.50 \pm 8.02$ & 0.005 \\
\hline
\end{tabular}

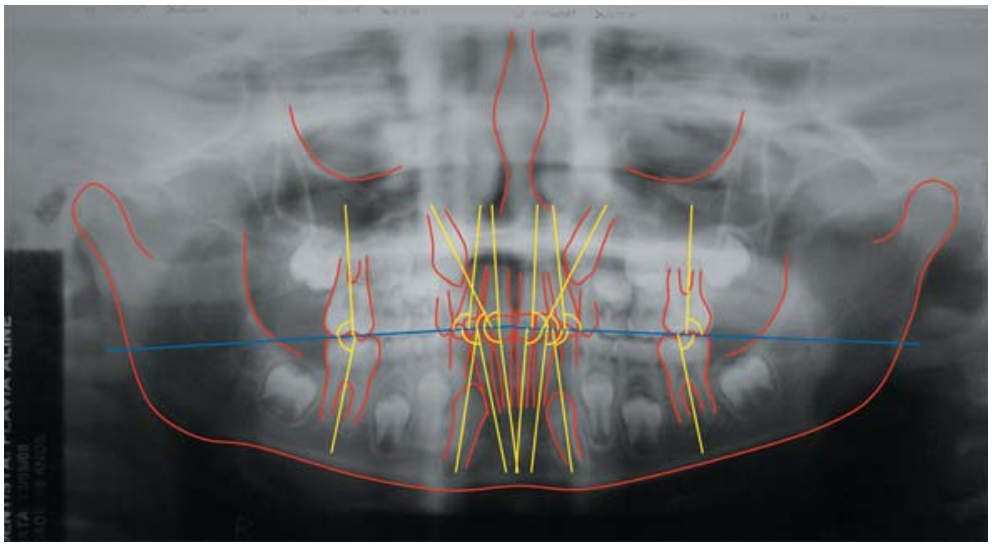

Figure 1- Panoramic radiograph showing the anatomic structures, the dental long axes and the angles measured relative to the reference line measured on the external side 


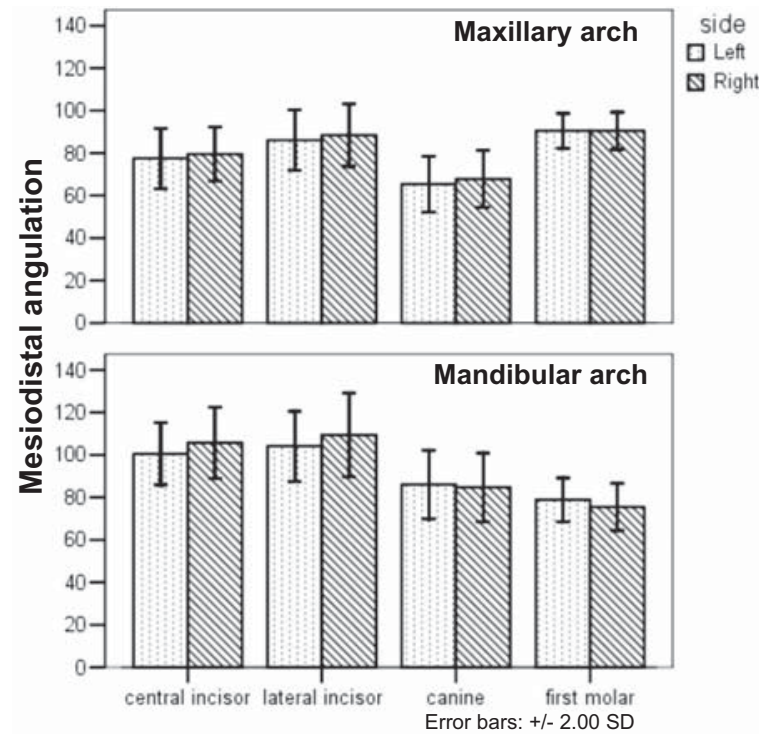

Figure 2- Mesiodistal angulation of differents homologous teeth in children with mixed dentition. $\mathrm{SD}=$ standard deviation

\section{DISCUSSION}

This study measured the mesiodistal root angulations of permanent incisors, canines and first molars among Brazilian children with normal occlusion in mixed dentition. A new horizontal reference line was used for measuring the tooth angulation.

The study of Ursi, et al. ${ }^{17}$ (1990) suggested using superior and inferior reference lines for the evaluation of the mesiodistal axial inclination in the panoramic radiographs of adults with permanent dentition, with the superior line passing through the lowest points of the right and left orbits and the inferior line through the centre of the right and the left mental foramina. Our study suggests using a reference line passing through the intercuspation mid-point of the permanent first molars and primary canines, to the right and the left sides, respectively. The choice was justified mainly by the difficulty of locating the mental foramen in the mixed dentition, which is small and superimposed by the tooth germs. Also, the permanent first molars and the primary canines are visible and easily identified structures, reducing the reference line errors.

The males showed angulations of 5 to 6 degrees greater than those of the females. Other studies report that differences of up to five degrees between the genders are clinically acceptable ${ }^{11,17}$. The alterations in the anterior mandibular area could be attributed to the panoramic radiograph distortions mentioned in the literature ${ }^{5,8}$.

The comparison of mesiodistal root angulations between the homologous permanent teeth showed statistically significant differences for the maxillary incisors and canine groups. Our results support the idea that asymmetrical eruption may be present in normal mixed dentition. Some studies ${ }^{7,14-16}$ report that there is no alignment pattern for the maxillary incisors, which may be protruded and present diastemas, which decreases the aesthetics, despite being a normal occlusion requiring no treatment.

Evaluation of the youngest (eight years old) and the oldest (ten years old) groups in the same mixed dentition revealed no differences. This could justify the absence of alterations in the mesiodistal angulation between these two groups, suggesting that no influence was established by canine eruption until that time.

Despite some inherent degree of distortion ${ }^{8}$, the panoramic radiograph is the most practical examination for evaluating tooth angulations. In the maxilla, however, the panoramic radiograph may lead to an overestimation of canine angulation ${ }^{4}$. The comparison of panoramic radiography with cone beam computed tomography revealed the anterior maxillary roots' over-inclination in a mesial direction and the posterior roots' over-inclination in a distal direction ${ }^{10}$. The greatest alteration occurred between the maxillary canines and the first premolars ${ }^{10}$. With regard to the anterior mandibular angulations, the authors ${ }^{10}$ reported that they were not bilaterally symmetric and the posterior roots tended to be mesially inclined. The results of our study also failed to show bilateral symmetry for the majority of the evaluated teeth.

The proposed reference line could be useful in mixed dentition root angulation evaluation. The normal values established for the root angulation of permanent teeth in the middle mixed dentition in Brazilian children may offer parameters for the early diagnosis of deviations, as well as signs of normal asymmetric pattern of eruption. This study determined the acceptable mean values in a homogeneous group, but longitudinal studies are welcome to confirm this normal behavior.

\section{CONCLUSIONS}

The following conclusions can be drawn: the proposed reference line could be useful in mixed dentition root angulation evaluation; there was a slight asymmetry in the mesiodistal angulation among homologous teeth, and also a small variation between the male and the female groups, but no difference between 8-and 10-year-old children.

\section{REFERENCES}

1- Al-Bitar ZB, Al-OmariI IK, Sonbol HN, Al-Amad HT, Hamdan AM. Mixed dentition analysis in a Jordanian population. Angle Orthod. 2008;78:670-5.

2- Broadbent $\mathrm{BH}$. The face of the normal child. Angle Orthod. $1937 ; 7: 183-208$.

3- Dahlberg G. Statistical methods for medical and biological students. London: George Allen \& Unwin; 1940. 
4- Fleming PS, Scott P, Heidari N, Dibiase AT. Influence of radiographic position of ectopic canines on the duration of orthodontic treatment. Angle Orthod. 2009;79:442-6.

5- Lucchesi MV, Wood RE, Nortjé CJ. Suitability of the panoramic radiograph for assessment of mesio-distal angulation of teeth in the buccal segments of the mandible. Am J Orthod Dentofacial Orthop. 1988;94:303-10.

6- Lundström A, Lundström F, Lebret LM, Moorees CF. Natural head orientation: basic considerations in cephalometric analysis and research. Eur J Orthod. 1995;17:111-20.

7- Moyers RE. Handbook of orthodontics. Chicago: Year Book; 1988.

8- Owens AM, Johal A. Near-end of treatment panoramic radiograph in the assessment of mesiodistal root angulation. Angle Orthod. 2008;78:475-81.

9- Paula S, Almeida AO, Lee PC. Prediction of mesiodistal diameter of unerupted lower canines and premolar using 45 degrees cephalometric radiography. Am J Orthod Dentofacial Orthop. 1995;107:309-14.

10- Peck JL, Sameshima GT, Miller A, Worth P, Hatcher DC. Mesiodistal root angulation using panoramic and cone beam CT. Angle Orthod. 2007;77:206-13.

11 - Samawi SSB, Burke PH. Angular distortion in the orthopantomogram. Br J Orthod. 1984;11:100-7.
12- Sangcharearn Y, Ho C. Maxillary incisor angulation and its effect on molar relationships. Angle Orthod. 2007;77:221-5.

13- Schindel RH, Duffy SL. Maxillary transverse discrepancies and potentially impacted maxillary canines in mixed-dentition patients. Angle Orthod. 2007;77:430-5.

14- Slaj M, Jezina MA, Lauc T, Rajić-Mestrović S, Miksić M. Longitudinal dental arch changes in the mixed dentition. Angle Orthod. 2003;73:509-14.

15- Tausche E, Luck O, Harzeer W. Prevalence of malocclusions in the early mixed dentition and orthodontic treatment need. Eur J Orthod. 2004;26:237-44.

16- Thilander B, Pena L, Infante C, Parada SS, Mayorga C. Prevalence of malocclusion and orthodontic treatment need in children and adolescents in Bogota, Colombia. An epidemiological study related to different stages of dental development. Eur J Orthod. 2001;23:153-67.

17- Ursi WJ, Almeida RR, Tavano O, Henriques JF. Assessment of mesiodistal axial inclination through panoramic radiography. J Clin Orthod. $1990 ; 24: 166-73$.

18- Warford JH Jr, Grandhi RK, Tira DE. Prediction of maxillary canine impaction using sectors and angular measurement. Am J Orthod Dentofacial Orthop. 2003;124:651-5. 